\title{
Evaluation of in vivo activation of protein kinase $A$ under non-dissociable conditions through the overexpression of wild-type and mutant regulatory subunits in Saccharomyces cerevisiae
}

\author{
Paula Portela, $\uparrow$ Vanina Zaremberg $†$ and Silvia Moreno
} Author for correspondence: Silvia Moreno. Tel: +54 114576 3342. Fax: + 541145763342.
e-mail: smoreno@qb.fcen.uba.ar

Departamento de Química Biológica, Facultad de Ciencias Exactas y Naturales, Universidad de Buenos Aires, Ciudad Universitaria, Pabellón 2, Piso 4, 1428 Buenos Aires, Argentina
BCY1-encoded protein kinase A (PKA) wild-type and mutant regulatory (R) subunits from Saccharomyces cerevisiae were inducibly overexpressed in their corresponding background strains containing the same mutation in the bcy1 gene. The aim of this approach was to shift the catalytic activity of PKA within the cell to the undissociated holoenzyme form(s) in order to evaluate whether the wild-type or the mutant forms of the holoenzyme could display catalytic activity. Two mutants of $R$ subunits were used: bcy1-16, with a complete deletion of CAMP-binding domain B; and bcy1-14, with a small deletion in the carboxy terminus of CAMP-binding domain A. Their overexpression caused an increase in the level of $R$ subunits in the range 40-90-fold, as detected by CAMP-binding activity, Coomasie-stained SDS-PAGE and Western blot analysis. The change in PKA activity attained by overexpression of $R$ was assessed in three ways: (i) through the analysis of PKA-dependent phenotypes, and (ii, iii) by measurement of PKA activity -l+ CAMP using the specific substrate kemptide in crude extracts (ii) and permeabilized cells (iii). Upon overexpression of the $R$ subunits, PKAdependent phenotypes were less severe when compared with their own background. However, a gradient in the degree of severity of phenotypes bcy1$14>b c y 1-16>$ wild-type was observed in the background strains and was maintained in the strains overexpressing the $R$ subunits. cAMP levels measured in background and in R-overexpressing strains showed an increase of around two orders accompanying the overexpression of the $R$ subunits. Three main conclusions could be drawn from the PKA activity measurements - I+ CAMP in crude extracts: (i) catalytic activity was not increased in compensation for the increase in $R$ subunits in any of the three cases (wild-type, bcy1-16 or bcy1-14 overexpression); (ii) PKA activity assayed in the absence of cAMP was lower in the case of extracts from strains overexpressing wild-type or bcy1-16 $R$ subunits when compared with the corresponding extracts without overexpression; and (iii) in these two cases, the great excess of $R$ subunits in the crude extracts displayed additional inhibitory capacity towards exogenously added catalytic (C) subunits. To provide an estimate of the in vivo activation of PKA, permeabilized cells from control strains and strains transformed with either wild-type, bcy1-16 or bcy1-14 R subunits were used to measure PKA activity in the presence of variable concentrations of CAMP. There were two main observations from the results: (i) the activity of PKA detected in the absence of exogenous CAMP was decreased in the strains overexpressing 


\section{the $\mathbf{R}$ subunits when compared to their corresponding backgrounds, and (ii) the sensitivity to activation by CAMP was decreased or almost nil. The biochemical and genetic results obtained are consistent with the hypothesis that within the cell it is possible to have catalytically active, cAMP-bound, undissociated PKA holoenzyme.}

Keywords: cAMP, holoenzyme, in situ activity, BCY1

\section{INTRODUCTION}

The eukaryotic cAMP-dependent protein kinases (PKAs) are tetramers composed of two regulatory (R) and two catalytic $(\mathrm{C})$ subunits. The holoenzyme $\left(\mathrm{R}_{2} \mathrm{C}_{2}\right)$ is catalytically inactive due to the blocking of the substrate site of $\mathrm{C}$ subunits with a pseudosubstrate site in the $\mathrm{R}$ subunits; contact through additional sites between $\mathrm{R}$ and $\mathrm{C}$ subunits yields an interacting affinity between them in the nanomolar range (Zhao et al., 1998). Activation results from allosteric interactions by which cAMP binding to two intrachain cAMP-binding sites (sites $\mathrm{A}$ and $\mathrm{B}$ ) in the $\mathrm{R}$ subunit decreases by five orders of magnitude its affinity for the $\mathrm{C}$ subunit (Døskeland et al., 1993). According to the dissociation constant of the mammalian holoenzymes with bound cAMP and the intracellular concentration of PKA in the micromolar range (Francis \& Corbin, 1994), cAMPsaturated PKA may exist within the cell without being dissociated into its subunits and it has been suggested that even cAMP-saturated PKA might retain some of the kinase activity of the free $\mathrm{C}$ subunit (Døskeland et al., 1993).

There are no adequate models at present suitable for explaining the variety of responses displayed by a system as a consequence of the action of different signals that are known to activate the same cAMP pathway (Taskén et al., 1997). The source of variability for selectively regulating different PKA phosphorylation events has been explained by the different affinity of the various isoenzymic forms of $\mathrm{R}$ subunit for cAMP and for $\mathrm{C}$ subunit isoforms, by substrate concentration, by enzyme localization through the interaction with anchoring proteins (AKAPs) and by crosstalk with other signal transduction pathways. A cAMP-bound holoenzyme with enzymic activity would add an interesting level of diversity to explain in vivo PKA behaviour.

We are interested in understanding the molecular mechanism for the activation of PKA in vivo, and have chosen as one of our models mutants of the Saccharomyces cerevisiae $\mathrm{R}$ subunit, isolated by Cannon et al. (1986) while looking for revertants of a characteristic ras 2 phenotype: lack of growth on non-fermentable carbon sources, a general nutritional defect associated with decreased PKA activity (Tatchell, 1986; Thevelein, 1992, 1994; Griffioen et al., 2000). The variant bcy1 alleles displayed different phenotypes. Many aspects of growth were affected, including growth on nonfermentable carbon sources, accumulation of glycogen, ability to sporulate and ability to survive starvation. None of the spontaneous mutations displayed a $b c y 1$ null phenotype. However the mutants could be placed into groups of increasing severity based on the number of traits affected (Cannon et al., 1990). In previous work using these mutants (Zaremberg \& Moreno, 1996), we have demonstrated that the degree of severity of the phenotypes in a ras 2 background, as well as the relative differences between the mutants, were maintained upon overexpression of the high-affinity cAMP phosphodiesterase (PDE2), while the severity of each mutant was decreased in a RAS2 background, although the differential gradient was still maintained. It is worth mentioning that the wild-type strain in a ras 2 background overexpressing PDE2 was not viable, as were the mutants with a disruption in the three genes encoding the C subunit of PKA (tpk1, tpk2, tpk3; Toda et al., 1987). These observations, together with some biochemical evidence, led us to conclude that the variant holoenzymes could respond to cAMP (RAS2 background) and that the difference in phenotypes displayed by the strains in the absence of cAMP (ras2 background plus overexpression of PDE2) could be explained by a differential constitutive activity of the mutated holoenzymes. According to our interpretation, the wild-type holoenzyme, in the absence of cAMP, could not display the minimum activity compatible with life, while the mutated forms of the enzyme could display differential constitutive activity in the absence of cAMP, probably due to a change in their tertiary structure in a way that resembled the conformational changes promoted by the interaction of cAMP with native $\mathrm{R}$ subunits and which differentially decreased the affinity of mutated R for C subunits.

In the present work, we attempted to produce within the cells undissociated wild-type and mutant holoenzymes by a different approach: overexpression of the corresponding $\mathrm{R}$ subunits, so as to shift the equilibrium of association/dissociation of PKA into its subunits towards the undissociated state. We chose to work with the wild-type strain and with two mutants displaying an intermediate and a severe phenotype, $b c y 1-16$ and $b c y 1$ 14, respectively (Zaremberg \& Moreno, 1996). The mutated $b c y 1-16$ allele has a complete deletion of cAMPbinding domain $\mathrm{B}$ of the $\mathrm{R}$ subunit, while the mutated bcy1-14 allele has a deletion in the carboxy-terminal part of cAMP-binding domain A, a region very recently discovered to be important in the interaction with the $\mathrm{C}$ subunit and in the cooperative interaction between the two cAMP-binding domains (Huang \& Taylor, 1998). 
Table 1. Lists of strains and nomenclature used

\begin{tabular}{|c|c|}
\hline Strain & Genotype \\
\hline $\begin{array}{l}\text { KT } 1115 \\
\text { KT } 1449 \\
\text { KT } 1451 \\
\text { S18 1D }\end{array}$ & $\begin{array}{l}\text { MAT a leu2 ura3 his3 pep } 4 \Delta \\
\text { MATa leu2 ura3 his3 pep } 4 \Delta \text { bcy1-16 } \\
\text { MAT } \text { leu2 ura3 his3 pep } 4 \Delta \text { bcy1-14 } \\
\text { MAT } \text { leu2 ura3 his3 trp1 ade8 tpk } 1^{\text {w1 }} \\
\text { tpk2:: HIS3 tpk3:: TRP1 }\end{array}$ \\
\hline Nomenclature & Description \\
\hline WT & KT $1115+$ YEp51 \\
\hline WTBCY & KT $1115+$ YEp51-BCY1 \\
\hline 16 & KT 1449+ YEp51 \\
\hline $16 b c y$ & KT $1449+$ YEp51-bcy1-16 \\
\hline 14 & KT $1451+$ YEp51 \\
\hline $14 b c y$ & KT $1451+$ YEp51-bcy1-14 \\
\hline
\end{tabular}

\section{METHODS}

Yeast strains and plasmids. The genotypes of the strains and the genetic nomenclature used in this study are listed in Table 1. The $b c y 1-14$ allele has a deletion (Gly-285 to Leu-298) in the carboxy-terminal part of cAMP-binding domain A, and bcy116 has an ochre mutation in Tyr-345, implicating a deletion of cAMP-binding site B (Cannon et al., 1990; Zaremberg \& Moreno, 1996).

To facilitate protein purification steps, $\Delta p e p 4$ strains containing the BCY1 or the $b c y 1-16$ and $b c y 1-14$ alleles were constructed by crossing each parental strain (MAT $\alpha$ leu 2 ura 3 bcy1 RAS2) to a MATa his3 $\Delta p e p 4$ strain, followed by isolation of leu2 ura3 his $3 \Delta$ pep 4 bcy 1 mitotic segregants.

The $2.9 \mathrm{~kb} B a m \mathrm{HI}-E c o \mathrm{RI}$ fragment including the BCY1 gene cloned in pBR322 was inserted into Bluescript SK(-) vector (Promega). A NcoI restriction site was then introduced by sitedirected mutagenesis (Kunkel et al., 1987) using the primer 5'GTAAGAATAAACCATGGTATCTTCT-3', which changes a $\mathrm{G}$ for a $\mathrm{C}$ one nucleotide before the first $B C Y 1$ codon. The $1.9 \mathrm{~kb} B s p$ EI-EcoRI fragment of $b c y 1-16$ and $b c y 1-14$ cloned in YEp24 (a generous gift from Kelly Tatchell, Louisiana State University, USA) containing the different mutations were exchanged for the corresponding fragment of the wild-type $B C Y 1$ (NcoI) subcloned in Bluescript SK $(-)$; in this way each mutant allele acquired the $\mathrm{NcoI}$ restriction site necessary for subcloning in the selected shuttle vector, YEp51. This vector is based on the yeast $2 \mu$ plasmid, which is typically present at 30-50 copies per cell and has an NcoI site after the GAL10 promoter, allowing the transcription of a cloned gene to be repressed by glucose and induced by galactose. The $2 \cdot 15 \mathrm{~kb}$ NcoI-HindIII fragment from SK-BCY1(NcoI), SK-bcy1$16(\mathrm{NcoI})$ and the $2 \cdot 10 \mathrm{~kb}$ fragment from $S K-b c y 1-14(\mathrm{NcoI})$ were inserted into YEp51 digested with the same enzymes, yielding the BCY1 gene and its mutated alleles under the control of the GAL10 promoter.

Overexpression of R subunits. Yeast strains containing wildtype or mutated alleles (bcy1-16 and bcy1-14) of the BCY1 gene were transformed by the lithium acetate method (Ito et al., 1983). LEU2 $2^{+}$transformants were selected. Overexpression was routinely checked before each experiment by measurement of cAMP-binding activity as described below. Untransformed strains and strains transformed with the empty vector or with the recombinant YEp51 vector expressed similar levels of PKA R subunit when grown in glucose.

Growth media. Yeast media were prepared as described by Sherman et al. (1981). Strains were grown on rich medium containing $2 \%$ Bactopeptone, $1 \%$ yeast extract and $2 \%$ galactose (YPgal) or $2 \%$ glucose (YPG). Synthetic media containing $0.67 \%$ yeast nitrogen base without amino acids, $2 \%$ glucose plus the necessary additions to fulfil auxotrophic requirements were used to maintain the selectable plasmids. Solid media contained $2 \%$ agar.

Phenotype analysis. Glycogen accumulation was detected by the brown colour produced by staining with iodine, analysed in situ by inversion of a plate over $\mathrm{I}_{2}$ crystals in a chromatography tank (Chester, 1968).

Respiration was measured by reduction of tetrazolium dyes; cells grown at $30{ }^{\circ} \mathrm{C}$ for $48 \mathrm{~h}$ were overlaid with $1.5 \%$ agar, $0 \cdot 1 \%$ tetrazolium/HCl. Plates were incubated at $30{ }^{\circ} \mathrm{C}$ for $1 \mathrm{~h}$ before photography. The formation of the red compound formazan correlates with respiration (Ogur et al., 1957).

Viability was assayed by inclusion of $20 \mathrm{mg}$ Phloxine B (Sigma) $1^{-1}$ in YPgal medium. Dead cells are stained by Phloxine B and show a pink colour (Fantes, 1981).

Heat shock resistance was determined after pregrowing yeast strains in rich medium (YPgal) to a concentration of $1 \times 10^{6}$ cells $\mathrm{ml}^{-1}$. Heat shock was performed in a water bath at $54^{\circ} \mathrm{C}$ for $15 \mathrm{~min}$. Serial dilutions of treated and untreated cells were spotted on YPgal plates and incubated for $48 \mathrm{~h}$ at $30^{\circ} \mathrm{C}$ before photography (Ma et al., 1999).

Crude extract preparation and partial purification of PKA. Yeast cells were grown to a concentration of $0.5-1.0 \times 10^{7}$ cells $\mathrm{ml}^{-1}$ (exponential phase) in medium YPgal. Cells (from $15 \mathrm{ml}$ cultures) were lysed by disruption with glass beads at $4{ }^{\circ} \mathrm{C}$ in $0.6 \mathrm{ml} 50 \mathrm{mM}$ potassium phosphate $\mathrm{pH} 6.8,5 \mathrm{mM}$ EDTA, $3 \mathrm{mM}$ EGTA, $120 \mathrm{mM} \mathrm{NaCl}, 10 \mathrm{mM}$ 2-mercaptoethanol, $1 \mathrm{mM}$ PMSF, $3 \mu \mathrm{g}$ antipain $\mathrm{ml}^{-1}, 1 \mathrm{mM}$ benzamidine. $\mathrm{HCl}$. Cell debris was pelleted by centrifugation for $5 \mathrm{~min}$ at $5000 \mathrm{~g}$ and the supernatant was used immediately for enzymic assays in crude extracts or subsequent partial purification.

PKA holoenzymes were partially purified by precipitation of the crude extracts with $1.7 \mathrm{M}\left(\mathrm{NH}_{4}\right)_{2} \mathrm{SO}_{4}$. Pellets were stored at $-20{ }^{\circ} \mathrm{C}$ and desalted by means of Sephadex G-25 filtration immediately before use. Protein was determined by the Bradford assay with BSA as standard.

Permeabilization procedure. This was performed according to Maggese et al. (1982). Cells $\left(5 \times 10^{8}\right)$ were suspended in $1 \mathrm{ml}$ $40 \mathrm{mM}$ Tris/HCl pH 7•4, 0.5 mM EDTA, $1 \mathrm{mM} \mathrm{PMSF,} 3 \mu \mathrm{g}$ antipain $\mathrm{ml}^{-1}, 1 \mathrm{mM}$ benzamidine. $\mathrm{HCl}$, mixed with $0 \cdot 15 \mathrm{ml}$ toluene/ethanol $(1: 4, \mathrm{v} / \mathrm{v})$ and vortexed for $5 \mathrm{~min}$. The cells were immediately pelleted, washed once and resuspended in the same buffer. Permeabilized cells were used within $30 \mathrm{~min}$ of preparation.

Determination of PKA subunit activities. The determination of $\mathrm{R}$ subunit activity was based on measurement of its binding capacity for $\left[{ }^{3} \mathrm{H}\right]$ cAMP. $\left[{ }^{3} \mathrm{H}\right] \mathrm{cAMP}$ was bound to free $\mathrm{R}$ subunits since addition of $\mathrm{NaCl}$ to the assay promoted complete dissociation of the holoenzyme. Under these conditions, complete exchange between endogenous bound cAMP and added $\left[{ }^{3} \mathrm{H}\right]$ cAMP was achieved by $30 \mathrm{~min}$ incubation at $30^{\circ} \mathrm{C}$.

In a standard assay, aliquots of partially purified samples (1-3 $\mu$ g protein) were incubated for $30 \mathrm{~min}$ at $30^{\circ} \mathrm{C}$ with $0 \cdot 3 \mu \mathrm{M} \quad\left[{ }^{3} \mathrm{H}\right]$ cAMP (55000 d.p.m. pmol $^{-1}$; DuPont New England Nuclear), $0.5 \mathrm{M} \mathrm{NaCl}, 1 \mathrm{mM}$ EDTA, $15 \mathrm{mM}$ Tris/ 
$\mathrm{HCl} \mathrm{pH} 7 \cdot 4$. Samples were filtered through nitrocellulose (MSI), as described by Moreno et al. (1983). cAMP-binding assays were linear with protein concentration.

C subunit activity was determined by assay of phosphotransferase activity with kemptide as a sustrate. The assay was started by mixing the samples (different protein amounts of each strain as indicated in Fig. 3) with assay mixture to give $50 \mathrm{mM}$ potassium phosphate $\mathrm{pH} 7 \cdot 5,200 \mu \mathrm{M}$ kemptide, $0 \cdot 1 \mathrm{mM}\left[\gamma^{32} \mathrm{P}\right]$ ATP (500 d.p.m. pmol $^{-1}$; DuPont New England Nuclear), $0 \cdot 1 \mathrm{mM}$ EGTA, $0 \cdot 1 \mathrm{mM}$ EDTA, $15 \mathrm{mM} \mathrm{MgCl}_{2}$, $10 \mathrm{mM} 2$-mercaptoethanol and $10 \mu \mathrm{M}$ cAMP (when added). After $30 \mathrm{~min}$ at $30^{\circ} \mathrm{C}$, aliquots were processed according to the phosphocellulose paper (P81; Whatman) method of Roskoski (1983). PKA assays were linear with time and protein concentration.

PKA assay in permeabilized cells was performed by incubation of samples containing $1 \cdot 2-2 \cdot 4 \times 10^{6}$ cells in a final volume of $70 \mu \mathrm{l}$ for $3 \mathrm{~min}$ at $30^{\circ} \mathrm{C}$ in the incubation mixture described above, except for the use of $0 \cdot 1 \mathrm{mM}\left[\gamma^{32} \mathrm{P}\right] \mathrm{ATP}$ of higher specific activity (1500 d.p.m. pmol ${ }^{-1}$ ) plus different concentrations of cAMP, as indicated in Fig. 4. PKA assays were linear with time and cell number.

PKA activity is expressed in units (U), defined as pmol $\mathrm{min}^{-1}$ of kemptide phosphorylated under the standard conditions.

Determination of cAMP levels. Samples containing $0 \cdot 1-1 \cdot 0 \mathrm{~g}$ cells (wet wt) were used for determination of cAMP, as described previously by Thevelein et al. (1987). A cAMP assay (kit supplied by Amersham) based on the competition of cAMP with $\left[{ }^{3} \mathrm{H}\right]$ cAMP for the $\mathrm{R}$ subunit of cAMP-dependent PKA, was used for all cAMP determinations.

Immunological analysis Samples of the partially purified preparations $(10 \mu \mathrm{g}$ per lane) were separated by SDS-PAGE, blotted onto nitrocellulose membranes (MSI) using $25 \mathrm{mM}$ Tris, $192 \mathrm{mM}$ glycine, $20 \%$ (v/v) methanol buffer in a Transphor apparatus (Hoefer). Blots were blocked with $5 \%$ non-fat milk, $0.05 \%$ Tween 20 in Tris-buffered saline (TBS), incubated with anti-BCY antibody [BCY1 (yN-19); Santa Cruz Biotechnology; diluted $1 / 500$ in blocking solution] overnight at $4{ }^{\circ} \mathrm{C}$. Blots were washed and incubated with antigoat IgG-horse radish peroxidase (Santa Cruz Biotechnology) for $1 \mathrm{~h}$ at room temperature. After incubation, blots were washed twice with blocking solution and twice with $0.05 \%$ Tween 20 in TBS. The membranes were incubated with Chemiluminescence Luminol Reagent (Santa Cruz Biotechnology) and immunoreactive bands were visualized by autoradiography.

\section{RESULTS AND DISCUSSION}

\section{Rationale of the approach}

Isogenic strains carrying the $w t, b c y 1-16$ and $b c y 1-14$ alleles of the BCY1 gene were transformed with YEp51 (LEU2) plasmid containing the corresponding $\mathrm{R}$ variants under the control of the GAL10 promoter. The rationale behind this approach was to shift the equilibrium of association/dissociation of the $\mathrm{R}$ and $\mathrm{C}$ subunits of PKA towards the associated state, so as to study the behaviour of the undissociated wild-type and mutated holoenzymes.

The response of the cells to the overexpression of the bcy1-16 and bcy1-14 alleles was particularly appealing since a previous work (Zaremberg \& Moreno, 1996) indicated that they could form holoenzymes with the $\mathrm{C}$ subunit and structural data derived from the crystallized

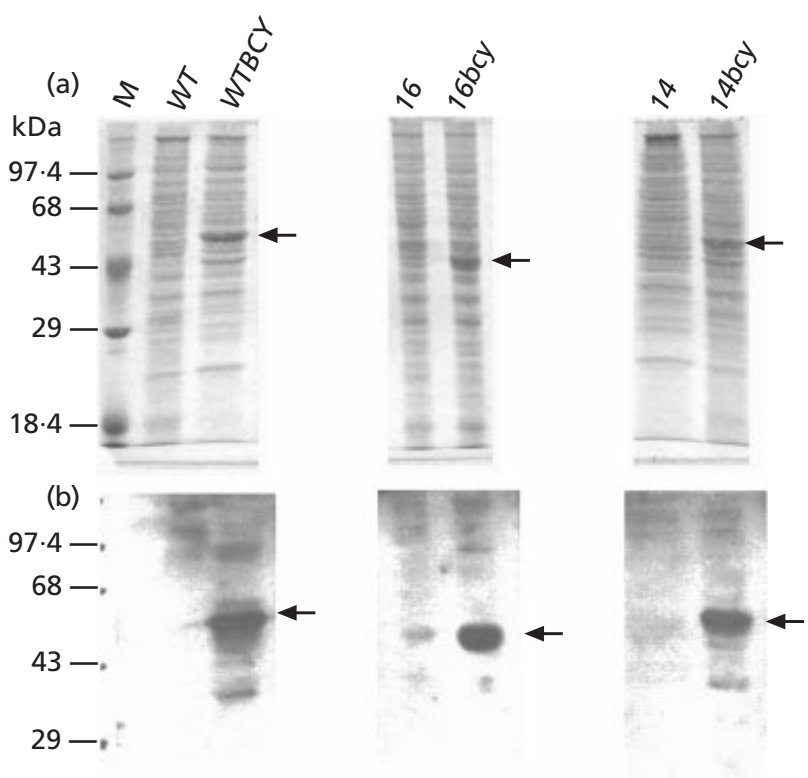

(c)

\begin{tabular}{|rcccccc|}
\hline Strain: & WT & WTBCY & 16 & $16 b c y$ & 14 & $14 b c y$ \\
\hline $\begin{array}{c}\text { fmol cAMP } \\
\text { bound }(\mu \mathrm{g} \\
\text { protein })^{-1}\end{array}$ & $1 \cdot 9 \pm 0 \cdot 6$ & $178 \cdot 2 \pm 2 \cdot 8$ & $2 \cdot 9 \pm 1 \cdot 3$ & $120 \pm 3$ & $1 \cdot 8 \pm 0 \cdot 9$ & $103 \pm 1 \cdot 6$ \\
\hline
\end{tabular}

Fig. 1. Analysis of the overexpression of $R$ subunits. (a) Gels stained with Coomassie brilliant blue following SDS-PAGE of partially purified samples of $R$ subunits. Equal amounts of proteins $(10 \mu \mathrm{g})$ were electrophoresed on a $10 \%$ polyacrylamide gel. Lane $\mathrm{M}$ contains molecular mass markers. Arrows on the right indicate wild-type and mutant $\mathrm{R}$ subunit proteins. (b) Immunoblot analysis. Gels equivalent to those in (a) were blotted and incubated with a commercial antibody against $R$ subunit as described in Methods. (c) $\left[{ }^{3} \mathrm{H}\right] \mathrm{CAMP}-$ binding activity. The reaction was performed as described in Methods. Values are means \pm SEM from two separate samples.

$\mathrm{R}$ subunit predicted that the principal defect of these mutated holoenzymes would be the lack of cooperativity in cAMP-binding, and therefore the activation would be less sensitive to cAMP. The $b c y 1-16$ allele has only one cAMP-binding site, and therefore no cooperativity could be expected, while the bcy1-14 mutant has a deletion in the $\alpha$-helix carboxy-terminal to cAMP-binding domain $\mathrm{A}$, reported to be essential in the cooperative interaction between both cAMP-binding sites A and $\mathrm{B}$, and in the interaction with the C subunit (Huang \& Taylor, 1998).

\section{Analysis of the overexpression of $\mathbf{R}$ subunit}

All the strains yielded $L E U^{+}$transformants able to grow in the presence of the inducer galactose. The level of overexpression of $\mathrm{R}$ subunits was analysed in transformants grown in YPgal media, using the same strains transformed with the empty vector as control. This analysis was performed by three methods: SDS-PAGE (Fig. 1a), Western blot (Fig. 1b) and $\left[\mathrm{H}^{3}\right]$ cAMP-binding activity (Fig. 1c).

Fig. 1(a) shows that the overexpression of the $\mathrm{R}$ subunit could be visualized by protein staining of samples 

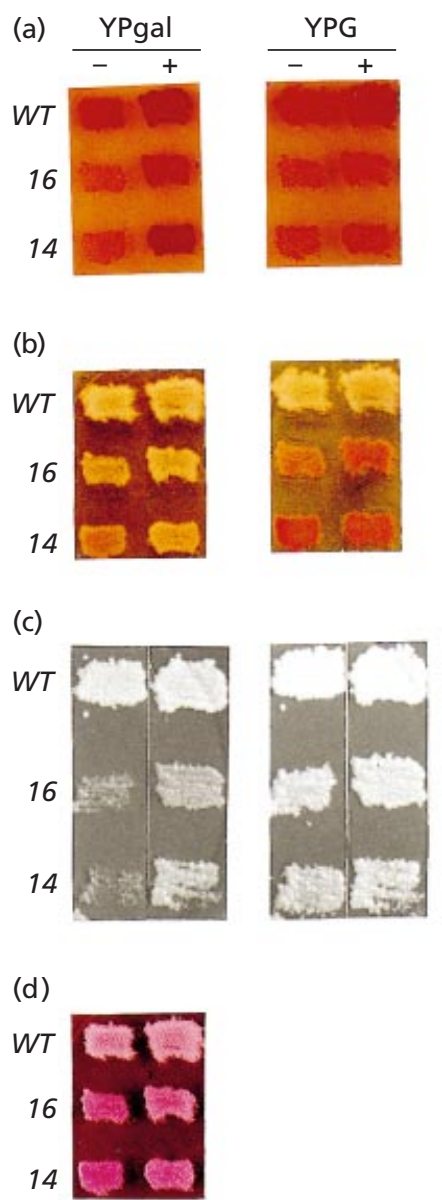

(e)
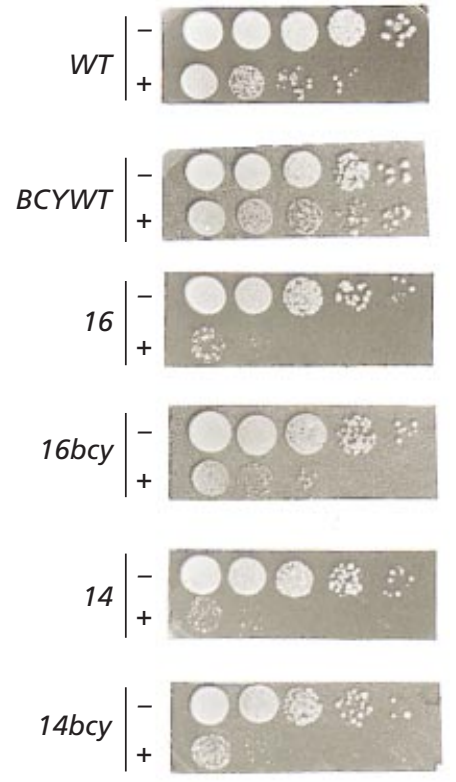

Fig. 2. Phenotypes of bcy1 mutants. (a-d) The strains indicated were spotted onto a master plate followed by replica plating. Strains transformed with the empty vector are indicated as $(-)$, while those transformed with the recombinant vectors are indicated as $(+)$. Each phenotype was analysed on galactose (YPgal), where the overexpression of $\mathrm{R}$ subunit takes place, and on glucose (YPG), as a control. (a) Growth on YPgal or YPG at $30^{\circ} \mathrm{C}$ for $48 \mathrm{~h}$ followed by staining with iodine vapour; (b) growth on YPgal or YPG at $30^{\circ} \mathrm{C}$ for $48 \mathrm{~h}$ followed by respiratory assay; (c) growth on YPgal or YPG at $37^{\circ} \mathrm{C}$ for $48 \mathrm{~h}$; (d) growth on YPgalPhloxine $\mathrm{B}$ at $30^{\circ} \mathrm{C}$ for $48 \mathrm{~h}$. (e) After a heat shock of $15 \mathrm{~min}$ at $54{ }^{\circ} \mathrm{C}$, the cell suspensions were diluted serially by a factor of 10 and spotted on YPgal plates $(+)$ together with their controls without heat shock $(-)$. Plates were incubated at $30^{\circ} \mathrm{C}$ for $48 \mathrm{~h}$. submitted to SDS-PAGE (comparing lane WT versus WTBCY, 14 versus $14 b c y$ and 16 versus $16 b c y$ ). The molecular masses of the overexpressed bands corresponded to those expected for the wild-type and mutated $\mathrm{R}$ subunit proteins. The apparent molecular masses were: $59 \cdot 3 \pm 0.9,48.7 \pm 0.9$ and $54.7 \pm 0.5 \mathrm{kDa}$ for $W T$, 16 and 14 , respectively (values are means \pm SEM from four independent gels).

Western blot analysis (Fig. 1b), confirmed that the overexpressed stained bands corresponded to the yeast $\mathrm{R}$ subunit protein, since they were detected with a specific anti-BCY1 antibody directed against the $\mathrm{NH}_{2}{ }^{-}$ terminal portion shared by the three proteins.

The amount of $\mathrm{R}$ subunit, monitored by measuring the cAMP-binding activity in partially purified extracts, also showed a large increase in expression in WTBCY, $16 b c y$ and $14 b c y$ as compared to their respective control cells. An overexpression of around 40-90-fold of active protein could be estimated for the three $\mathrm{R}$ variants (Fig. 1c).

\section{Phenotypes associated with the PKA pathway}

Depletion of cAMP or inactivation of PKA causes yeast cells to arrest proliferation and to enter permanently into the stationary phase, G0. When the cells enter G0, they acquire all the characteristics typical of stationary phase, for example strong accumulation of trehalose and glycogen, high stress resistance, strong expression of a variety of genes controlled by STRE elements in their promoters, and constitutive sporulation even in rich medium (Thevelein \& de Winde, 1999).

To investigate the consequences of overexpressing the $\mathrm{R}$ subunits, we analysed some phenotypes associated with the PKA pathway. The phenotypes analysed were glycogen accumulation, respiratory capacity, growth at $37^{\circ} \mathrm{C}$ on galactose, viability and heat-shock resistance (Fig. 2). It has previously been reported for these strains that the range of severity, either in ras 2 or in RAS2 backgrounds and taking into account several phenotypes, is $w t<b c y 1-16<b c y 1-14$ (Zaremberg \& Moreno, 1996).

Overexpression of the respective $\mathrm{R}$ subunit in each strain produced hyperaccumulation of glycogen and increased heat-shock resistance (Fig. 2a, e).

The ability to grow on galactose at $37^{\circ} \mathrm{C}$ (Fig. 2c) gradually decreased in the order $W T>16>14$, the last strain being unable to use this carbon source at this temperature. However, the overexpression of the respective $\mathrm{R}$ subunit allowed the growth of this strain under these conditions.

Viability analysis was assayed by staining dead cells with Phloxine B. Fig. 2(d) shows that control back- 
ground strains had a higher intensity of pink when comparing WT to 14 and that the overexpression of $\mathrm{R}$ subunit produced in all cases a decrease in the shade of pink, which is interpreted as an increase in viability. The $14 b c y$ strains were least viable when compared to $B C Y W T$ and to $16 b c y$.

The ability to reduce triphenyltetrazolium (pink-red colour) for testing the respiratory capacity of the parental ras 2 strains on different carbon sources has been analysed previously (Cannon et al., 1986). In this study, we analysed this phenotype on glucose and galactose media for the selected RAS2 strains overexpressing or not overexpressing the $\mathrm{R}$ subunit. It is interesting to note that $b c y 1-16$ and $b c y 1-14$ strains in a RAS2 background present a pink-red colour when growing on glucose in contrast with their ras 2 counterparts (Fig. 2b and Cannon et al., 1986). The colour is stronger for $b c y 1-14$ and increases on galactose. Overexpression of the $\mathrm{R}$ subunit partially reversed the increase in respiratory capacity observed on galactose (see Fig. 2b, YPgal plate).

Summarizing and integrating the results of Fig. 2, we can conclude that the overexpression of $\mathrm{R}$ subunits, observed only when cells were grown in YPgal, promoted in every case a shift in each of the phenotypes to one correlating with lower PKA activity. However, an important difference among the three strains was still observed and the order of severity of the phenotypes was in all cases maintained. The order of severity of the phenotypes suggests that mutants 16 and 14 could display higher PKA activity than their wild-type counterparts, even when we expected that in the presence of high levels of $\mathrm{R}$ subunit all the PKA would be as an undissociated holoenzyme.

In previous work (Zaremberg \& Moreno, 1996) in which the strategy used in order to obtain undissociated holoenzyme was to lower cAMP levels by overexpression of the high-affinity cAMP phosphodiesterase gene (PDE2) in ras 2 backgrounds, the $B C Y 1$ strain was not viable, as if the undissociated holoenzyme could not display the minimum of activity necessary for survival in the absence of cAMP. It is worth pointing out that with the approach used in this work we obtained a viable WT strain overexpressing high levels of $\mathrm{R}$ subunit, indicating that these cells have a PKA activity level compatible with life.

\section{cAMP levels}

cAMP accumulation in yeast is strongly inhibited by PKA, since mutants with reduced activity of this enzyme display hyperaccumulation of cAMP, while mutants with unbridled PKA activity display reduced levels of cAMP (Nikawa et al., 1987). Pde1 (cAMP phosphodiesterase) has been identified as a possible target of the feedback inhibition mechanism involved in control of agonist-induced cAMP signalling, while Pde2 specifically controls the basal cAMP level in the cell (Ma et al., 1999).
To evaluate whether the differences in PKA activity of the mutant and wild-type strains affected the feedback inhibition of cAMP, we measured the cAMP levels for each strain grown in YPgal medium. The levels of cAMP for WT, 16 and 14 were 0.06, 0.02 and 0.01 pmol $(\mathrm{mg} \text { wet } \mathrm{wt})^{-1}$, respectively. These results are consistent with the phenotypic analysis, in the sense that the mutants have phenotypes, in this case the cAMP level, correlating with the different level of PKA activity they display.

However we did not attempt to evaluate the feedback inhibition of cAMP in the strains overexpressing $\mathrm{R}$ subunits, since an increase in cAMP levels matching the increase in the R subunit was expected. In fact, when the levels of cAMP were measured in those strains an increase of around two orders was observed [WTBCY, $4 \mathrm{pmol}\left(\mathrm{mg}\right.$ wet $\left.\mathrm{wt}^{-1}\right) ; 16 b c y, 6 \mathrm{pmol}(\mathrm{mg} \text { wet } \mathrm{wt})^{-1}$; and $\left.14 b c y, 6 \mathrm{pmol}(\mathrm{mg} \text { wet } \mathrm{wt})^{-1}\right]$. These values are in the range of those found in the PKA-attenuated strain S18 1D grown in the same medium (data not shown). This strain has a deletion in the tpk2 and in the tpk3 genes, and the $t p k 1$ gene has a weak phenotype $\left(t p k 1^{\mathrm{wk}}\right)$, with no detectable PKA activity in crude extracts. It has been reported that this mutant is impaired in the rigorous feedback mechanism controlling cAMP levels, and shows an increase in the accumulation of the cyclic nucleotide of around 1000-fold (Nikawa et al., 1987). However, our interpretation is that the great increase in cAMP levels in the strains overexpressing the $\mathrm{R}$ subunits is not due to very low levels of PKA activity, as in the S18 $1 \mathrm{D}$ strain, but to the fact that the excess of $\mathrm{R}$ subunits sequester CAMP, making it insensitive to degradation by cAMP phosphodiesterases. It is well known that the affinity of R subunits towards cAMP is in the range of one order greater than the one displayed by the highaffinity phosphodiesterases. An increase in cAMP levels upon overexpression of recombinant $\mathrm{R}$ subunit has previously been described in Escherichia coli (Saraswat et al., 1986).

The accuracy of these determinations and the difference in the methodologies applied to measure cAMP level and cAMP-binding activity do not permit assessment of whether there is an excess of cAMP over R subunit, or if there is some cAMP-free $\mathrm{R}$ subunit within the cells.

\section{In vitro measurement of PKA activity}

We were interested in studying whether there was a compensating increase in $\mathrm{C}$ subunit levels upon overexpression of the $\mathrm{R}$ subunit. Preliminary results suggested that at least the levels of $T \mathrm{pk} 1 \mathrm{p}$, as detected by Western blot with antibodies against this isoform, were not increased (data not shown). From the literature we knew that strains carrying only TPK1 or TPK 2 genes have half of the total kinase activity detected in the wildtype strain (Mazón et al., 1993), and that disruption of any one of the three TPK genes did not produce a change in the levels of mRNA from the other two genes (Robertson et al., 2000), showing that cells do not compensate for the loss of one $\mathrm{C}$ subunit protein by 
(a)
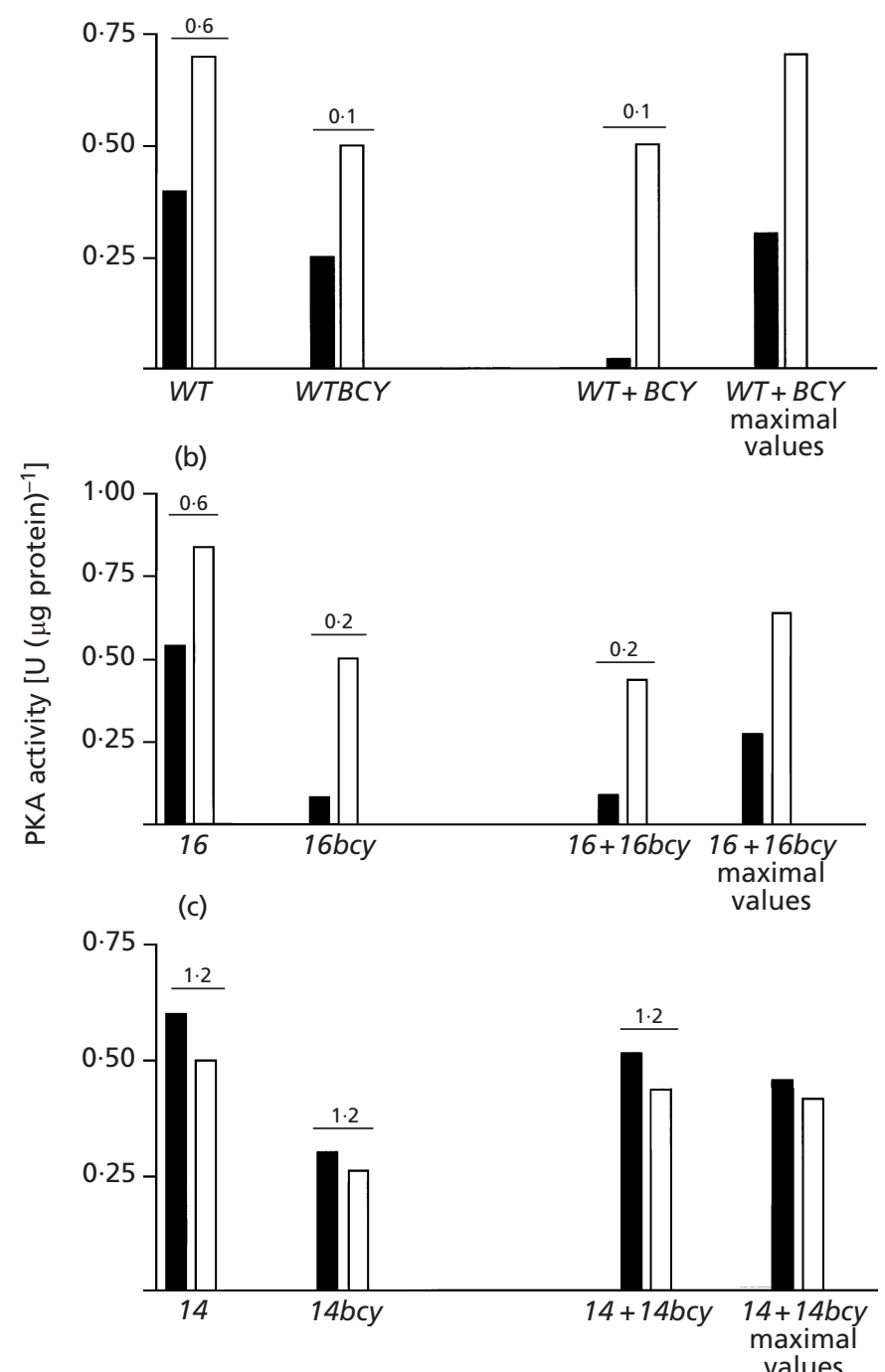

Fig. 3. PKA activity in crude extracts. PKA activity was measured in aliquots of crude extracts of WT and WTBCY (a), 16 and $16 \mathrm{bcy}$ (b), and 14 and 14bcy (c), under the standard assay conditions, in the absence (black bars) or in the presence (white bars) of $10 \mu \mathrm{M}$ cAMP. Activities are expressed in $\mathrm{U}$ ( $\mu \mathrm{g}$ total protein) ${ }^{-1}$. The values over each pair of bars represent the ratio $-/+$ CAMP. The third pair of bars in each panel show activity measured in a sample containing a mixture of equal amounts of protein of crude extracts from background and overexpressing strains. The fourth pair of bars in each panel show means of the individual activities $(-/+$ CAMP) of each crude extract contributing to the mixture, derived from the experiments shown by the first and second pair of bars. overexpression of another. So we could reasonably suppose that since Tpk1p levels did not change, the total levels of $\mathrm{C}$ subunit would also remain constant. However, we undertook the measurement of PKA activity in crude extracts from background strains and from strains overexpressing $\mathrm{R}$ subunit in order to evaluate this parameter more carefully. Fig. 3 summarizes these results; the first two pairs of bars in each panel show the activity of the background strains and from the strains overexpressing $\mathrm{R}$ subunit, measured in the absence and in the presence of cAMP. The white bars, representing the activity in the crude extracts measured in the presence of $10 \mu \mathrm{M}$ cAMP, are a measure of the total levels of catalytic activity present in the cell (either in the form of holoenzyme or in the form of free $\mathrm{C}$ subunit). In none of the cases was there an increase in catalytic activity compensating for the overexpression of the $\mathrm{R}$ subunit. To the contrary, the levels in the WTBCY1, $16 b c y$ and $14 b c y$ strains were $50-70 \%$ of the levels of their corresponding backgrounds.
The degree of activation of PKA by cAMP in crude extracts does not reflect the degree of activation in intact cells, since the dilution imposed by the extract plus the one in the assay produces a shift in the association/ dissociation equilibrium towards the dissociated state, even in the absence of added cAMP. This is particularly evident in the case of $S$. cerevisiae PKA, since the affinity in the interaction between $\mathrm{R}$ and $\mathrm{C}$ subunits is not as high as that in higher eukaryotic PKAs. Depending on the aliquot used for the PKA assay, it is common to observe in the case of wild-type PKA an activity almost independent from cAMP or slightly dependent. For example, the first pair of bars show a $-/+$ cAMP ratio of 0.6 for the WT and the 16 enzymes and complete independence for the 14 enzyme. However it is quite remarkable that a very low $-/+$ cAMP ratio was observed in the in vitro assay upon overexpression of $\mathrm{R}$ subunit in the cases of WTBCY and 16bcy, indicating that the excess of $\mathrm{R}$ subunit shifted the association/ dissociation equilibrium to the holoenzyme state of the 

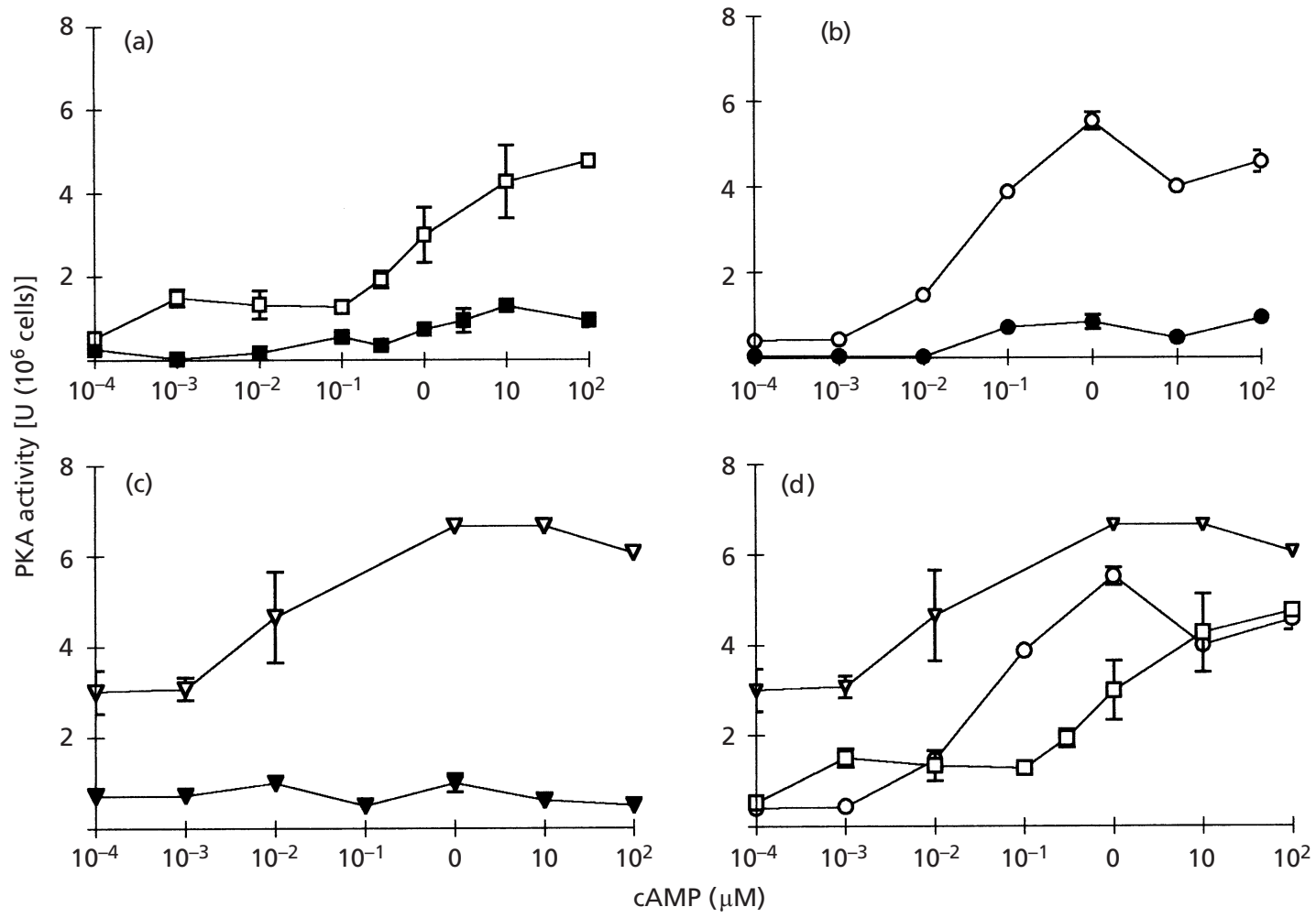

Fig. 4. PKA activity in permeabilized cells. PKA activity was measured in the presence of increasing concentrations of cAMP in permeabilized cells of $W T(\square)$ and WTBCY $(\square)$ strains (a), $16(\bigcirc)$ and $16 b c y(0)$ strains (b), and $14(\nabla)$ and $14 b c y$ $(\nabla)$ strains (c) under the standard conditions described in Methods. Panel (d) shows the activation of PKA by cAMP in the background strains without overexpression of the respective $R$ subunits, redrawn from panels (a), (b) and (c) for comparative purposes. Values are means \pm SEM from two independent samples. In all cases, the PKA activity in the absence of cAMP was identical to that in the presence of $10^{-4} \mu \mathrm{M}$ cAMP.

PKA. To analyse whether in the extracts from the WTBCY and $16 b c y$ strains there was extra inhibitory capacity derived from the excess of $\mathrm{R}$ subunit, we performed the experiment shown by the third pair of bars in Fig. 3, in which we mixed aliquots containing equal amounts of protein from extracts from background strains and from strains overexpressing $\mathrm{R}$ subunit. The fourth pair of bars is a theoretical representation of the maximal values that could be expected from the mixture of the extracts (estimated through the mean of the activities of each extract in the mixture) and is shown to facilitate the interpretation of the results. The experimental results shown in the third pair of bars show that the activity ratio $-/+$ cAMP of the mixture is identical to the one obtained with the extracts from WTBCY or $16 b c y$ strains. These low ratios indicate that the excess of $\mathrm{R}$ subunit (provided by the $W T B C Y$ or $16 b c y$ extracts in the mixture) is enough to shift the equilibrium association/dissociation of the holoenzyme to the undissociated state, even upon the addition of an extra dose of $\mathrm{C}$ subunit (provided by the WT or 16 extracts).

In the case of the extracts derived from the 14 and from the $14 b c y$ strains, no cAMP dependence could be observed in any of the in vitro assays. However, we know from previous results using permeabilized cells from the 14 strain that the PKA from this mutant is dependent on cAMP (Zaremberg \& Moreno, 1996, and see Fig. 4 above). We believe that with this mutant, and in the in vitro conditions, the equilibrium association/ dissociation is shifted towards dissociation, even under excess of $\mathrm{R}$ subunit.

\section{PKA activity in permeabilized cells}

To have an insight on the stage of activation of PKA in vivo when overexpressing the $\mathrm{R}$ subunits, we assayed this enzymic activity in permeabilized cells. This method is a good estimate of in vivo activity since PKA localization and concentration, as well as the interaction with other proteins and with cAMP, are still maintained. Fig. 4 shows the results of kemptide phosphorylation by PKA in permeabilized cells incubated with different concentrations of cAMP. Two principal conclusions can be derived from the results of Fig. $4(\mathrm{a}, \mathrm{b}$ and $\mathrm{c})$ : in the case of the cells overexpressing the $\mathrm{R}$ subunit, the activity in the absence of cAMP, or at very low concentration of the nucleotide, is lower than in the corresponding background strain; and the response to activation by cAMP is very poor or there is no response, as in the case of the $14 b c y$ cells. It is worth noting that 
the maximum activity obtained at $100 \mu \mathrm{M}$ cAMP in WTBCY, 16bcy and $14 b c y$ cells is around $10-20 \%$ of their corresponding backgrounds at the same cAMP concentration. However, from the results of Fig. 3 we know that the extracts derived from those cells are capable of expressing a total activity that is not smaller than $50-70 \%$ of their corresponding background controls. These results are consistent with published results and theoretical kinetic predictions showing that the apparent activation constant of cAMP for PKA increases when the ratio R/C is increased (Schwechheimer \& Hofmann, 1977; Houge et al., 1990). For example, in in vitro assays using type I PKA, the $K_{\mathrm{A}}$ for cAMP was increased fivefold when the ratio $\mathrm{R} / \mathrm{C}$ was increased fourfold (Schwechheimer \& Hofmann, 1977). It is not surprising that in our case, where the ratio $\mathrm{R} / \mathrm{C}$ was increased in the order of 40-90-fold, the holoenzyme, as measured in permeabilized cells, was not fully activated by (WTBCY and 16bcy) or almost insensitive to $(14 b c y)$ cAMP, at least up to $100 \mu \mathrm{M}$. Higher concentrations of cAMP were not assayed since they interfere with the PKA assay through competitive inhibition with ATP.

It is interesting to compare the response to cAMP displayed by the holoenzymes in the permeabilized cells from the background strains WT, 16 and 14 (Fig. 4d). The phosphorylation of the exogenous substrate, kemptide, at low cAMP concentration and even in the absence of added cAMP, was similar for WT and for 16, whereas it was higher for 14 . The maximum activity attained in the three cases was quite similar. However, the behaviour of the three strains in response to cAMP in the range of physiologically relevant concentrations $(0 \cdot 01-1 \cdot 00 \mu \mathrm{M})$ (Londesborough \& Lukkari, 1980) is very different. The activity of PKA in the 14 strain cells is particularly high at a wide range of cAMP concentrations, while the activity in cells derived from the 16 strain surpasses that of the wild-type cells, particularly in the range $0 \cdot 1-1 \cdot 0 \mu \mathrm{M}$.

\section{Integration of the results}

Our principal aim is to understand the in vivo activation of PKA. In this work, we attempted to produce undissociated wild-type and mutant holoenzymes within the cells by overexpressing the corresponding R subunits (wild-type, bcy1-16 and bcy1-14 alleles), so as to shift the equilibrium of association/dissociation of PKA towards the undissociated state. Our rationale was that if the undissociated holoenzymes could express PKA activity, we would still find a gradient in the relative phenotypes displayed by the different strains.

The overexpression of active $\mathrm{R}$ subunits turned out to be very efficient in the three cases (Fig. 1); the catalytic activity did not increase concomitantly, as detected by the in vitro assays in crude extracts in the presence of cAMP (Fig. 3); consequently, an increase in the R/C ratio of around two orders was achieved. The levels of cAMP, when overexpressing the R subunits, were largely increased; comparing the levels of cAMP obtained in the overexpressing strains with the total cAMP-binding capacity in those cells, we can estimate that at least most of the excess of $\mathrm{R}$ is in the cAMP-bound form. Even though the affinity of cAMP-bound $\mathrm{R}$ subunit towards the $\mathrm{C}$ subunit is much lower than the one of free $\mathrm{R}$ subunit, we could still expect a shift in the equilibrium of association/dissociation of the holoenzyme towards the undissociated state. The results of the in vitro measurement of PKA activity for the WTBCY and 16bcy extracts (Fig. 3) suggest that the $\mathrm{C}$ subunit is in the undissociated state, bound to $\mathrm{R}$. The fact that these two holoenzymes (containing the wild-type and the bcy1-16 alleles) could be maintained in an undissociated form in crude extracts caused us to predict that within the cells, where the enzyme concentration is much higher and the dissociation processes are therefore much more unfavourable, it would be more probable to find $\mathrm{C}$ subunit bound to $\mathrm{R}$. We have estimated that the enzyme concentration in permeabilized cells (Fig. 4) is around 300-fold higher that in the in vitro assays (Fig. 3), taking into account the volume of the cells, the volume of the crude extracts and the dilution during the enzymic assay.

From the results shown in Fig. 3(c) we could not predict whether the holoenzyme containing the bcy1-14 allele would also be within the cell mostly in an undissociated state when overexpressing the $b c y 1-14 \mathrm{R}$ subunit, since the measured activity was always independent of cAMP. In permeabilized cells (Fig. 4c) we observed a PKA activity also independent from cAMP, but with ten-fold lower activity than that of the background strain assayed in the presence of cAMP. However, from the in vitro assays we know that the total catalytic activity of $14 b c y$ is around $50 \%$ of the level of the background strain 14 . These results are interpreted as being the consequence of having an undissociated, partially active, mutated holoenzyme within the cell, which cannot be activated by cAMP, in the assayed range of concentration, due to the excess of the mutated $\mathrm{R}$ subunit. We believe that the bcy1-14 allele, due to the deletion of the $\alpha$ helix $\mathrm{C}$ of cAMP-binding domain A, has lost the cooperative effect in the activation of the holoenzyme by cAMP.

A very interesting observation is the fact that the WTBCY cells are viable. In our previous work (Zaremberg \& Moreno, 1996) we showed that wild-type strains in a ras 2 background were not viable when the high-affinity cAMP phosphodiesterase was overexpressed. This means that the undissociated holoenzyme in the absence of cAMP has no activity. However, the viability of the WTBCY cells, in which the $C$ subunit is in the undissociated state, as $R_{2} C_{2}$ or cAMP $\mathrm{R}_{2} \mathrm{C}_{2}$ species, suggests that the cells display at least a minimun of activity of PKA, which we think can be attributed to the cAMP-bound holoenzyme.

The shift of the phenotypes of each strain to a less severe one when its corresponding $\mathrm{R}$ subunit is overexpressed (Fig. 2) can be easily explained by a decrease in the PKA activity. It is more difficult to explain why the strains overexpressing the $\mathrm{R}$ subunit maintain the same order of severity $(14 b c y>16 b c y>W T B C Y)$ than the background strains $(14>16>W T)$, whether those strains 
have a RAS2 or a ras 2 background. We can envisage two non-exclusive interpretations for these results. The changes in the in vivo PKA activity and in the phenotypes are due to a real shift in the equilibrium towards the holoenzyme form of PKA; however the detected PKA activity reflects either (i) the small percentage of free $\mathrm{C}$ in the system or (ii) active holoenzyme, probably bound to cAMP. Although the first possibility cannot be excluded, principally in the case of the $14 b c y$ strain, we favour the second because from the results of Figs 3 and 4 we think that, under overexpression, all of the catalytic activity in the three cases is bound to R, either in the form of $\mathrm{R}_{2} \mathrm{C}_{2}$ or cAMP $\mathrm{R}_{2} \mathrm{C}_{2}$. More biochemical work will have to be undertaken in order to conclusively explain the difference in behaviour of the wild-type and mutated holoenzymes.

\section{ACKNOWLEDGEMENTS}

We are very grateful to Kelly Tatchell for inviting V.Z. to his laboratory to construct the YEp-51 recombinant plasmids and the $b c y 1$ pep $4 \Delta$ strains under the supervision of Mashid Company, to whom we are also very grateful. We acknowledge the gift of the S18 1D strain to Stephen Garrett. This work was supported by grants from the University of Buenos Aires, Agencia Nacional de Promoción Científica y Tecnológica, Consejo Nacional de Investigaciones Científicas y Técnicas and Ministerio de Salud through the fellowship Carrillo-Oñativia. During the course of this work P.P and V.Z were graduate fellows of University of Buenos Aires.

\section{REFERENCES}

Cannon, J. F., Gibbs, J. B. \& Tatchell, K. (1986). Suppressors of the ras 2 mutation of Saccharomyces cerevisiae. Genetics 113, 247-264.

Cannon, J. F., Gitan, R. \& Tatchell, K. (1990). Yeast cAMPdependent protein kinase regulatory subunit mutations display a variety of phenotypes. J Biol Chem 265, 11897-11904.

Chester, V. (1968). Heritable glycogen storage deficiency in yeast and its induction by ultraviolet light. J Gen Microbiol 51, 49-56.

Døskeland, S. O., Maronde, E. \& Gjersten, B. T. (1993). The genetic subtypes of cAMP-dependent protein kinase-functionally different or redundant? Biochim Biophys Acta 1178, 249-258.

Fantes, P. A. (1981). Isolation of cell size mutants of a fission yeast by a new selective method: characterization of mutants and implications for division control mechanisms. J Bacteriol 146, 746-754.

Francis, S. H. \& Corbin, J. D. (1994). Structure and function of cyclic nucleotide dependent protein kinases. Annu Rev Physiol 56, 237-272.

Griffioen, G., Anghileri, P., Imre, E., Baroni, M. D. \& Ruis, H. (2000). Nutritional control of nucleocytoplasmic localization of cAMP-dependent protein kinase catalytic and regulatory subunits in Saccharomyces cerevisiae. J Biol Chem 275, 1449-1456.

Houge, G., Vintermyr, O. K. \& Døskeland, S. O. (1990). The expression of cAMP-dependent protein kinase subunits in primary rat hepatocyte cultures. Cyclic AMP down-regulates its own effector system by decreasing the amount of catalytic subunit and increasing the mRNAs for the inhibitory (R) subunits of cAMP-dependent protein kinase. Mol Endocrinol 4, 481-488.
Huang, L. J. \& Taylor, S. S. (1998). Dissecting cAMP binding domain $\mathrm{A}$ in the RI $\alpha$ subunit of cAMP-dependent protein kinase. Distinct subsites for recognition of cAMP and the catalytic subunit. J Biol Chem 273, 26739-26746.

Ito, H., Fukuda, Y., Murata, K. \& Kimura, A. (1983). Transformation of intact yeast cells treated with alkali cations. $J$ Bacteriol 153, 163-168.

Kunkel, T. A., Roberts, J. D. \& Zakour, R. A. (1987). Rapid and efficient site-specific mutagenesis without phenotypic selection. Methods Enzymol 154, 367-382.

Londesborough, J. \& Lukkari, T. M. (1980). The $\mathrm{pH}$ and temperature dependence of the activity of the high $K_{\mathrm{m}}$ cyclic nucleotide phosphodiesterase of baker's yeast. J Biol Chem 255, 9262-9267.

Ma, P., Wera, S., Van Dijck, P. \& Thevelein, J. M. (1999). The PDE1-encoded low-affinity phosphodiesterase in the yeast Saccharomyces cerevisiae has a specific function in controlling agonist-induced cAMP signaling. Mol Biol Cell 10, 91-104.

Maggese, M. C., Galvagno, M. A., Cantore, M. L. \& Passeron, S. (1982). In situ measurement of cAMP related enzymes in the dimorphic fungus Mucor rouxii. Cell Biol Int Rep 6, 1101-1108.

Mazón, M. J., Beherens, M. M., Morgado, E. \& Portillo, F. (1993). Low activity of the yeast cAMP-dependent protein kinase catalytic subunit TPK3 is due to the poor expression of the TPK3 gene. Eur J Biochem 213, 501-506.

Moreno, S., Pastori, R. \& Passeron, S. (1983). Protein kinase of Mucor rouxii. Unshielding of new cyclic AMP-binding sites upon dissociation of the ternary complex holoenzyme-cyclic AMP. Mol Cell Biochem 52, 13-16.

Nikawa, J., Cameron, S., Toda, T., Ferguson, K. \& Wigler, M. (1987). Rigorous feed back control of cAMP levels in Saccharomyces cerevisiae. Genes Dev 1, 931-937.

Ogur, M., St John, R. \& Nagai, S. (1957). Tetrazolium overlay technique for population studies of respiration deficiency in yeast. Science 125, 982-991.

Robertson, L. S., Causton, H. C., Young, R. A. \& Fink, G. R. (2000). The yeast A kinases differentially regulate iron uptake and respiratory function. Proc Natl Acad Sci U S A 97, 5984-5988.

Roskoski, R. (1983). Assay of protein kinase. Methods Enzymol 99, 3-6.

Saraswat, L. D., Filutowicz, M. \& Taylor, S. S. (1986). Expression of the type I regulatory subunit of cAMP-dependent protein kinase in Escherichia coli. J Biol Chem 261, 11091-11096.

Schwechheimer, K. \& Hofmann, F. (1977). Properties of regulatory subunit of cyclic AMP-dependent protein kinase (peak I) from rabbit skeletal muscle prepared by urea treatment of the holoenzyme. J Biol Chem 252, 7690-7696.

Sherman, F., Fink, G. \& Hicks, J. B. (1981). Methods in Yeast Genetics : a Laboratory Manual. Cold Spring Harbor, NY: Cold Spring Harbor Laboratory.

Taskén, K., Skålhegg, B. S., Taskén, K. A. \& 13 other authors (1997). Structure, function, and regulation of human cAMPdependent protein kinases. Adv Second Messengers Phosphoprotein Res 31, 191-204.

Tatchell, K. (1986). RAS genes and growth control in Saccharomyces cerevisiae. J Bacteriol 166, 364-367.

Thevelein, J. M. (1992). The RAS-adenylate cyclase pathway and cell cycle control in Saccharomyces cerevisiae. Antonie Leeuwenhoek 62, 109-130.

Thevelein, J. M. (1994). Signal transduction in yeast. Yeast 10, 1753-1790. 
Thevelein, J. M. \& de Winde, J.H. (1999). Novel sensing mechanisms and targets for the cAMP-protein kinase A pathway in the yeast Saccharomyces cerevisiae. Mol Microbiol 33, 904-918.

Thevelein, J. M., Beullens, M., Honshoven, F., Hoebeeck, G., Detremerie, K., Den Hollander, J. A. \& Jans, A. W. A. (1987). Regulation of the cAMP levels in the yeast Saccharomyces cerevisiae: intracellular $\mathrm{pH}$ and the effect of membrane depolarizing compounds. J Gen Microbiol 133, 2191-2196.

Toda, T., Cameron, S., Sass, P., Zoller, M. \& Wigler, M. (1987). Three different genes in Saccharomyces cerevisiae encode the catalytic subunits of the cAMP-dependent protein kinase. Cell $\mathbf{5 0}$ 277-287.
Zaremberg, V. \& Moreno, S. (1996). Analysis of the mechanism of activation of cAMP-dependent protein kinase through the study of mutants of yeast regulatory subunit. Eur J Biochem 237, 136-142.

Zhao, J., Hoye, E., Boylan, S., Walsh, D. A. \& Trewhella, J. (1998). Quaternary structures of a catalytic subunit-regulatory subunit dimeric complex and the holoenzyme of the cAMP-dependent protein kinase by neutron contrast variation. J Biol Chem 273, 30448-30459.

Received 1 September 2000; revised 5 December 2000; accepted 15 January 2001. 